\title{
A Case of Extra-axial Cavernous Angioma Originating from the Oculomotor Nerve
}

\author{
Toshitaka Inui ${ }^{1}$ Hiroyuki Hashimoto ${ }^{1}$ Kenta Fujimoto ${ }^{1}$ Tateo Shimokawara ${ }^{1}$ Mitsuhisa Nishiguchi ${ }^{1}$ \\ Syohei Yokoyama ${ }^{1}$ Koji Omoto ${ }^{1}$ Ryuta Matsuoka ${ }^{1}$ Takahide Yaegaki ${ }^{1}$ \\ ${ }^{1}$ Department of Neurosurgery, Osaka General Medical Center, \\ Osaka, Japan \\ Address for correspondence Toshitaka Inui, MD, Department of \\ Neurosurgery, Osaka General Medical Center, 3-1-56 Bandai-Higashi, \\ Sumiyoshi-ku, Osaka 558-8558, Japan (e-mail: t-inui@gh.opho.jp).
} Indian J Neurosurg 2016;5:133-135.
Abstract Keywords
- cavernous angioma
- extra-axial
- oculomotor nerve
- magnetic resonance imaging

We report a case of extra-axial cavernous angioma, which originated from the oculomotor nerve and, by hemorrhaging, resulted in oculomotor nerve palsy. Cavernous angioma generally occurs in the brain parenchyma. Therefore, this case is extremely rare. We believe that preoperative sequential magnetic resonance imaging contributed to preoperative diagnosis in this case.

\section{Introduction}

The prevalence of cavernous angioma (CA) in the central nervous system is considered to be approximately 0.3 to $0.5 \%$, and most CAs of the central nervous system develop within the brain parenchyma, such as within the cerebral hemisphere, the brainstem, or the basal ganglia. ${ }^{1}$ In rare cases, extra-axial CAs have been located in the cavernous sinus and internal auditory meatus. We encountered a case of CA that originated from the oculomotor nerve and led to oculomotor nerve palsy because of bleeding.

\section{Case Report}

A 51-year-old man presented with double vision, right ptosis, and mydriasis. Magnetic resonance imaging (MRI) revealed an approximately $1-\mathrm{cm}$ circle-shaped lesion located around the right cavernous sinus in the extraparenchymal area (-Fig. 1A). The lesion had a mirror-image appearance, which suggested an internal hemorrhage. This round mass showed a slight enhancement, and its mirror image changed over time ( - Fig. 1B, C). A cerebral angiogram revealed no abnormal findings. Because the patient's symptoms were caused by this lesion, we planned a craniotomy to remove the mass.

A normal right frontotemporal craniotomy was performed. The mass was attached to the anterior petroclinoid ligament, distinct from the parenchyma, and was covered with a membrane. The contents of the mass including bleeding were confirmed through the membrane (-Fig. 2A). After the membrane was punctured and the hematoma was absorbed, the membrane of the posterior part was dissected, and we recognized that pressure had been exerted to the outside rear of the oculomotor nerve. The epineurium of the oculomotor nerve and the outer membrane of the mass were partly continuous (-Fig. $\mathbf{2 B}$ ). The membrane was resected such that the normal structure of the oculomotor nerve was completely retained. In histopathologic examination, CA was diagnosed. During the 3 days after the operation, the patient's oculomotor palsy gradually improved. Three months after the operation, MRI showed that the mass had disappeared completely ( - Fig. $\mathbf{3}$ ). Although slight diplopia and mydriasis remained, the ocular movement disorder and ptosis had disappeared completely.

\section{Discussion}

Most CAs originates from intra-axial brain parenchyma, and reports of CA from the extra-axial brain tissue are very rare. Previous studies on CAs originating from the cranial nerve report 33 optic nerve or facial nerve cases, 32 auditory nerve cases, and 7 third cranial nerve cases, as well as fewer cases received

December 9, 2015

accepted

February 10, 2016

published online

July 14, 2016
DOI http://dx.doi.org/

10.1055/s-0036-1584593. ISSN 2277-954X.
(C) 2016 Neurological Surgeons' Society of India
License terms

(이요 $\Theta \circledast$ 


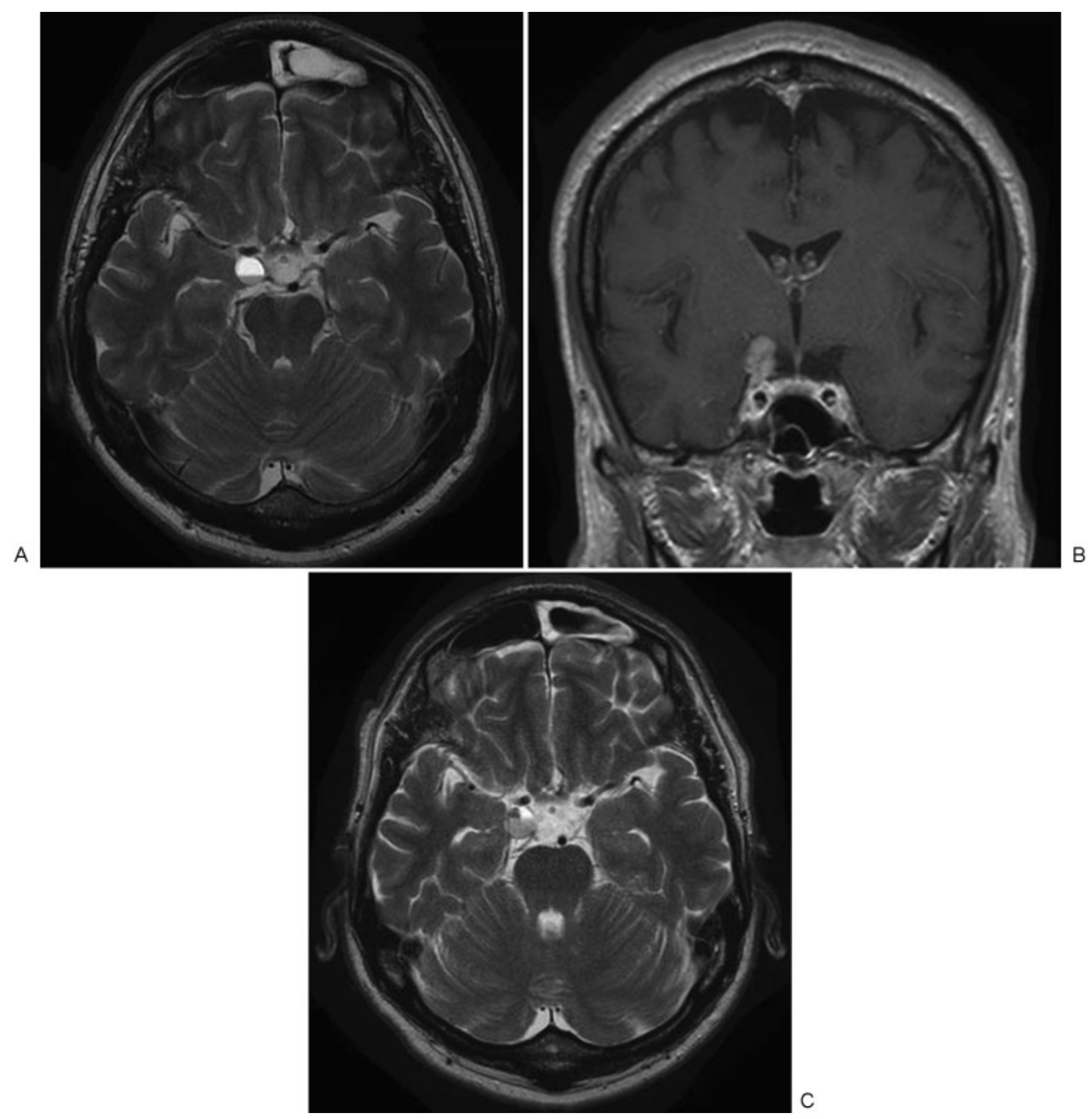

Fig. 1 (A) T2-weighted MRI image (axial view) revealing an extraparenchymal lesion that presented with a mirror image around the right cavernous sinus; (B) gadolinium-enhanced MRI image (coronal view) showing a slightly enhanced mass in the right cavernous sinus; (C) followup T2-weighted MRI image (axial view) obtained 1 week later. The extraparenchymal lesion now has a mosaic pattern around the right cavernous sinus.

at other locations. ${ }^{2-5}$ Five of seven previously reported cases involved rapid onset of oculomotor nerve palsy, similar to our case. All symptoms result from bleeding. There is no known sex difference in the prevalence of intracranial CA as a whole; however, angiomas that originate from facial nerves tend to be more common among men, and angiomas that develop from the middle cranial fossa tend to be more common among women. CAs originating from the oculomotor nerve have been reported in five men and two women, thus tending to be more frequent among men.

Because CA that develops from the oculomotor nerve is extremely rare, preoperative differential diagnosis is difficult. In the present case, the changes observed in sequential MRI may have been characteristic of CA. Specifically, the low- and the high-signal portion presented as mirror images in a T2-weighted image at the first medical examination, but the image had evolved into a mosaic form in a later MRI image. Therefore, we believe that some of the bleeding moved over time. It has been reported that ultrahigh intensity in a T2-weighted image is relatively characteristic of an extra-axial $\mathrm{CA}^{6,7}$ and the findings in the present case are consistent with this suggestion.

For extra-axial CAs, surgical methods and functional outcomes differ substantially according to the originating 


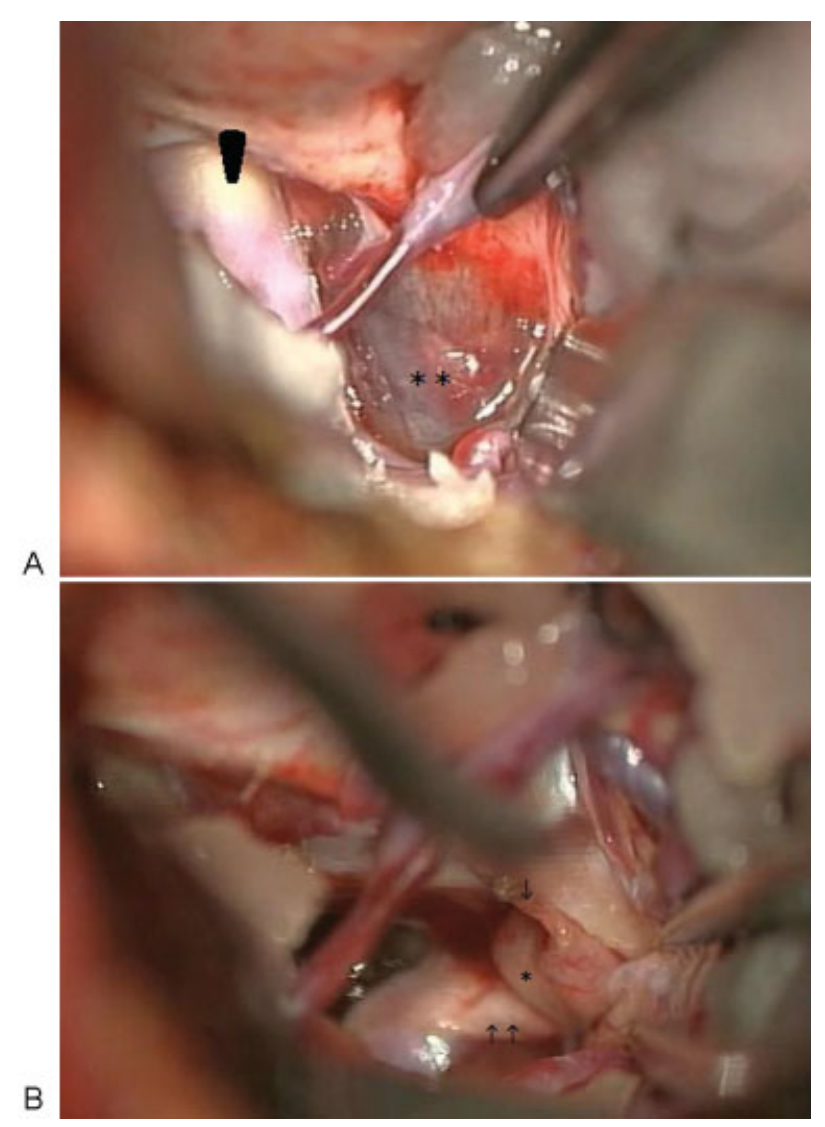

Fig. 2 (A) Intraoperative photograph showing the extraparenchymal lesion behind the right internal carotid artery. (B) Intraoperative photograph demonstrating the relation of the oculomotor nerve and the lesion after resection of the cavernous angioma. Arrow, resection point from the cavernous angioma; arrowhead, right internal carotid artery; asterisk, right oculomotor nerve; double arrow, right superior cerebellar artery; double asterisk, cavernous angioma.

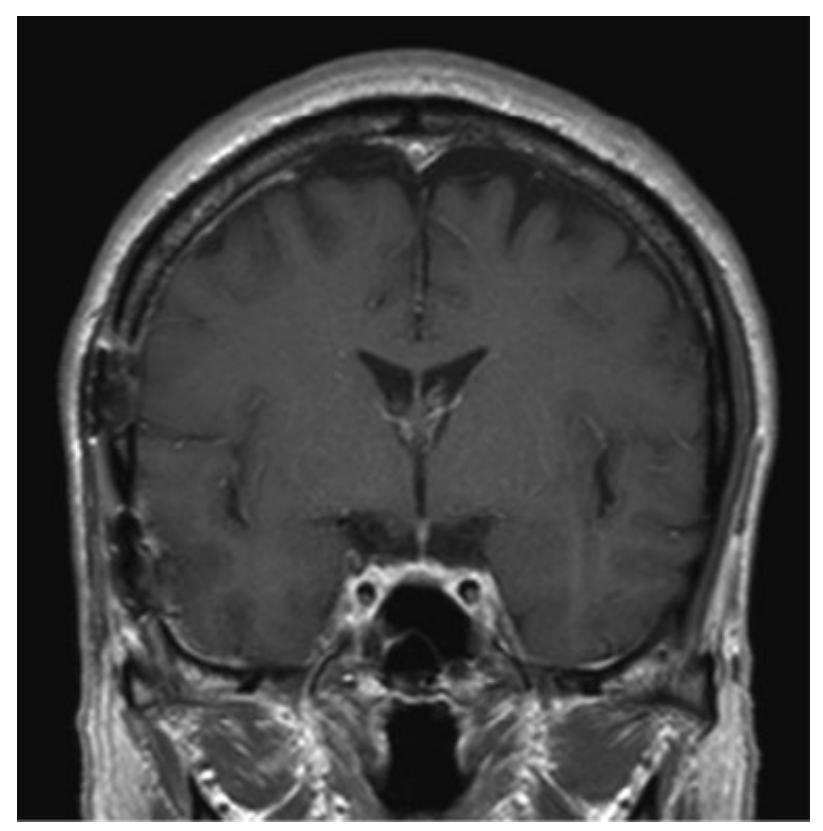

Fig. 3 Gadolinium-enhanced MRI image (coronal view) 3 months after the operation. tissue. ${ }^{7,8}$ CAs originating from the dura mater of the internal auditory meatus and skull base are perfused from the capillary plexus. For such cases, it has been suggested that there is a relatively low risk of postoperative nerve palsy because the adhesion to the nerve is weak. In contrast, CAs that develop from the cranial nerve are perfused from the vascular plexus of the nerve and are continuous with the epineurium. For such cases, nerve palsy shows only slight improvement postoperatively, specifically because detachment from the normal nerve is difficult. An incision along the major axis of the nerve fiber and piecemeal resection of the hematoma have been proposed as means of retaining neurologic function. ${ }^{5}$ In the present case, we resected the internal hematoma in a piecemeal fashion and confirmed the continuity with the oculomotor nerve after internal decompression. We believe that our intraoperative neuroprotective approach resulted in a good functional outcome.

\section{Conclusion}

We have reported a case of CA that originated from the oculomotor nerve and, by hemorrhaging, resulted in oculomotor nerve palsy. This type of CA is extremely rare, but we believe that CA should be considered during differential diagnosis. In addition, we suggest that the exact origin of the angioma is related to postoperative functional outcomes.

Conflict of Interest

None.

\section{References}

1 Kondziolka D, Lunsford LD, Kestle JR. The natural history of cerebral cavernous malformations. J Neurosurg 1995;83(5): 820-824

2 Itshayek E, Perez-Sanchez X, Cohen JE, Umansky F, Spektor S. Cavernous hemangioma of the third cranial nerve: case report. Neurosurgery 2007;61(3):E653, discussion E653

3 Ogilvy CS, Pakzaban P, Lee JM. Oculomotor nerve cavernous angioma in a patient with Roberts syndrome. Surg Neurol 1993; 40(1):39-42

4 Wolfe SQ, Manzano G, Langer DJ, Morcos JJ. Cavernous malformation of the oculomotor nerve mimicking a partially thrombosed posterior communicating artery aneurysm: report of two cases. Neurosurgery 2011;69(2):E470-E474

5 Scott RM. Oculomotor nerve cavernous angioma in a patient with Robert's syndrome [letter]. Surg Neurol 1994;41(4):352

6 Linskey ME, Sekhar LN. Cavernous sinus hemangiomas: a series, a review, and an hypothesis. Neurosurgery 1992; 30(1):101-108

7 Sasaki T, Sasaki T, Okamoto K, Ishida T, Kirino T. Cavernous angioma of the internal acoustic meatus-case report. Neurol Med Chir (Tokyo) 1999;39(12):847-851

8 Adachi K, Yoshida K, Akiyama T, Kawase T. Cavernous angioma of the vestibular nerve: case report and literature review. Surg Neurol 2008;70(1):82-86, discussion 86 